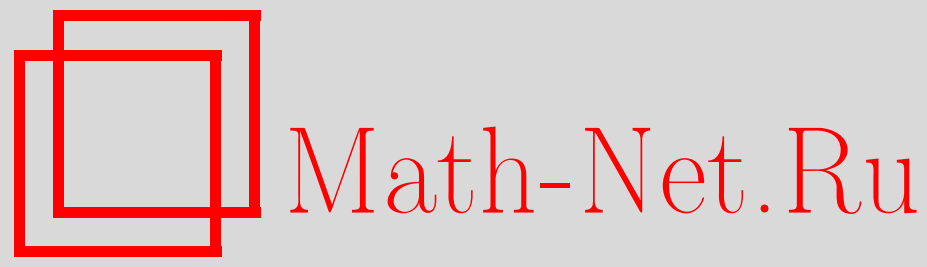

А. В. Соболев, Квазиклассическая асимптотика псевдодифференциальных операторов с разрывными символами: гипотеза Видома, Функи. анализ и его прил., 2010, том 44, выпуск 4, 86-90

DOI: https://doi.org/10.4213/faa3013

Использование Общероссийского математического портала MathNet.Ru подразумевает, что вы прочитали и согласны с пользовательским соглашением

http://www.mathnet.ru/rus/agreement

Параметры загрузки:

IP : 3.80 .181 .102

26 апреля 2023 г., 15:42:28




492-508. [20] V. Kostrykin, K. Makarov, Proc. Amer. Math. Soc., 136:6 (2008), 20672071. [21] М. Г. Крейн, Матем. сб., 33:3 (1953), 597-626. [22] И. М. Лифшиц, ЖЭТФ, 17 (1947), 1076-1089. [23] И. М. Лифшиц, Успехи матем. наук, 7:1 (1952), 171-180. [24] A. Pushnitski, Bull. London Math. Soc., 40:2 (2008), 227-238. [25] A. Pushnitski, Ann. Henri Poincaré, 10:4 (2009), 793-822. [26] A. Pushnitski, D. Yafaev, J. Funct. Anal., 259:8 (2010), 1950-1973. [27] A. Pushnitski, The Birman-Schwinger principle on the essential spectrum, готовится к печати. [28] J. Robbin, D. Salamon, Bull. London Math. Soc., 27:1 (1995), 1-33. [29] A. V. Sobolev, Ann. Inst. H. Poincaré Phys. Théor., 58:1 (1993), 55-83. [30] Д. Р. Яфаев, Математическая теория рассеяния, Изд-во С.-Петербургского университета, С.-Петербург, 1994.

Department of Mathematics, King's College London e-mail: alexander.pushnitski@kcl.ac.uk

Поступило в редакцию 13 января 2010 г.

УДК 517.984 .42

\title{
Квазиклассическая асимптотика псевдодифференциальных операторов с разрывными символами: гипотеза Видома
}

\author{
(C) 2010. А. В. Соболев
}

Памяти Михаила Шлемовича Бирмана

1. Для (комплекснозначного) символа $a=a(\mathbf{x}, \boldsymbol{\xi}), \mathbf{x}, \boldsymbol{\xi} \in \mathbb{R}^{d}, d \geqslant 1$, и произвольной функции $u$ из класса Шварца введем в $L^{2}\left(\mathbb{R}^{d}\right)$ псевдодифференциальный оператор

$$
\left(\mathrm{Op}_{\alpha} a\right) u(\mathbf{x})=\left(\frac{\alpha}{2 \pi}\right)^{d} \int e^{i \alpha(\mathbf{x}-\mathbf{y}) \boldsymbol{\xi}} a(\mathbf{x}, \boldsymbol{\xi}) u(\mathbf{y}) d \mathbf{y} d \boldsymbol{\xi}
$$

зависящий от большого параметра $\alpha>0$. Пусть $\Lambda, \Omega-$ две области в $\mathbb{R}^{d}$ и $\chi_{\Lambda}(\mathbf{x}), \chi_{\Omega}(\boldsymbol{\xi})$ - их индикаторы. Мы используем обозначение $P_{\Omega, \alpha}=\mathrm{Op}_{\alpha}\left(\chi_{\Omega}\right)$. В настоящей заметке изучаются оператор

$$
T_{\alpha}(a)=T_{\alpha}(a ; \Lambda, \Omega)=\chi_{\Lambda} P_{\Omega, \alpha} \mathrm{Op}_{\alpha}(a) P_{\Omega, \alpha} \chi_{\Lambda}
$$

и его симметризованный вариант

$$
S_{\alpha}(a)=S_{\alpha}(a ; \Lambda, \Omega)=\chi_{\Lambda} P_{\Omega, \alpha} \operatorname{Re} \mathrm{Op}_{\alpha}(a) P_{\Omega, \alpha} \chi_{\Lambda} .
$$

Нас интересует двучленная асимптотика следов $\operatorname{tr} g\left(T_{\alpha}\right), \operatorname{tr} g\left(S_{\alpha}\right)$ при $\alpha \rightarrow \infty$ с подходящей гладкой функцией $g$, такой, что $g(0)=0$. Введем асимптотические коэффициенты

$$
\begin{gathered}
\mathfrak{W}_{0}(a)=\frac{1}{(2 \pi)^{d}} \int_{\Lambda} \int_{\Omega} a(\mathbf{x}, \boldsymbol{\xi}) d \boldsymbol{\xi} d \mathbf{x} \\
\mathfrak{W}_{1}(g ; a)=\frac{1}{(2 \pi)^{d-1}} \int_{\partial \Lambda} \int_{\partial \Omega} \mathfrak{A}(g ; a(\mathbf{x}, \boldsymbol{\xi}))\left|\mathbf{n}_{\partial \Lambda}(\mathbf{x}) \cdot \mathbf{n}_{\partial \Omega}(\boldsymbol{\xi})\right| d S_{\boldsymbol{\xi}} d S_{\mathbf{x}}
\end{gathered}
$$


где $\mathbf{n}_{\partial \Lambda}(\mathbf{x})$ и $\mathbf{n}_{\partial \Omega}(\boldsymbol{\xi})$ - единичные векторы внешних нормалей к границам $\partial \Lambda$ и $\partial \Omega$ в точках х и $\boldsymbol{\xi}$ соответственно и

$$
\mathfrak{A}(g ; b)=\frac{1}{(2 \pi)^{2}} \int_{0}^{1} \frac{g(b t)-t g(b)}{t(1-t)} d t .
$$

Коэффициент $\mathfrak{A}(g ; b)$ корректно определен для любого комплексного (вещественного) числа $b$, если $g$ является $C^{1}$-функцией комплексной (вещественной) переменной.

Цель работы - установить двучленные асимптотические формулы, приведенные в следующей теореме.

Теорема 1. Пусть $\Lambda, \Omega \subset \mathbb{R}^{d}, d \geqslant 2$, - ограниченные области в $\mathbb{R}^{d} c$ $C^{1}$ - и $C^{3}$-границами соответственно, и пусть $a \in C_{0}^{2(d+2)}\left(\mathbb{R}^{d} \times \mathbb{R}^{d}\right)$.

(1) Пусть g аналитична в круге радиуса $R>0$ и $g(0)=0$. Если $R$ достаточно велик, то

$$
\operatorname{tr} g\left(T_{\alpha}(a)\right)=\alpha^{d} \mathfrak{W}_{0}(g(a))+\alpha^{d-1} \log \alpha \mathfrak{W}_{1}(g ; a)+o\left(\alpha^{d-1} \log \alpha\right) \quad \text { nрu } \alpha \rightarrow \infty .
$$

(2) Пусть $g \in C^{\infty}(\mathbb{R})$ u $g(0)=0$. Тогда след $\operatorname{tr} g\left(S_{\alpha}(a)\right)$ удовлетворяет асимптотической формуле (6), в которой символ а заменен на $\operatorname{Re} a$.

Было бы естественно ожидать, что переменные $\mathbf{x}$ и $\boldsymbol{\xi}$ в операторе $T_{\alpha}(a)$ равноправны. В самом деле, в работе [20, с. 173] с помощью элементарных вычислений было показано, что $\mathbf{x}$ и $\boldsymbol{\xi}$ можно поменять местами. Вместе с тем условия на $\Lambda$ и $\Omega$ в теореме 1 явно асимметричны. В настоящее время неясно, как исправить этот недостаток.

Теорема 1 позволяет вывести асимптотические формулы для функции распределения дискретного спектра оператора $S_{\alpha}(a)$, т. е. для числа его собственных значений на интервале $I=\left(\lambda_{1}, \lambda_{2}\right)$, где $\lambda_{1}<\lambda_{2}$ и $\lambda_{1} \lambda_{2}>0$ :

$$
n\left(\lambda_{1}, \lambda_{2} ; \alpha\right)=\operatorname{tr} \chi_{I}\left(S_{\alpha}(a)\right) .
$$

Следствие 2. Пусть выполнены условия теоремы 1. Предположим также, что $a_{-}<a(\mathbf{x}, \boldsymbol{\xi})<a_{+}$для всех $\mathbf{x} \in \Lambda, \boldsymbol{\xi} \in \Omega$ с некоторьми $a_{-}>0, a_{+}<\infty$. Eсли $\left[a_{-}, a_{+}\right] \subset I$, mo

$$
n\left(\lambda_{1}, \lambda_{2} ; \alpha\right)=\left(\frac{\alpha}{2 \pi}\right)^{d}|\Lambda||\Omega|+\alpha^{d-1} \log \alpha \mathfrak{W}_{1}\left(\chi_{I} ; a\right)+o\left(\alpha^{d-1} \log \alpha\right),
$$

где коэффичиент $\mathfrak{W}_{1}\left(\chi_{I} ; a\right)$ определен в (4) и

$$
\mathfrak{A}\left(\chi_{I} ; a(\mathbf{x}, \boldsymbol{\xi})\right)=\frac{1}{(2 \pi)^{2}} \log \left(\frac{a(\mathbf{x}, \boldsymbol{\xi})}{\lambda_{1}}-1\right) \quad \text { nрu } \alpha \rightarrow \infty .
$$

Eсли $\left[\lambda_{1}, \lambda_{2}\right] \subset\left(0, a_{-}\right)$, mo

$$
n\left(\lambda_{1}, \lambda_{2} ; \alpha\right)=\alpha^{d-1} \log \alpha \mathfrak{W}_{1}\left(\chi_{I} ; a\right)+o\left(\alpha^{d-1} \log \alpha\right),
$$

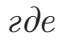

$$
\mathfrak{A}\left(\chi_{I} ; a(\mathbf{x}, \boldsymbol{\xi})\right)=\frac{1}{(2 \pi)^{2}} \log \frac{\lambda_{2}\left(a(\mathbf{x}, \boldsymbol{\xi})-\lambda_{1}\right)}{\lambda_{1}\left(a(\mathbf{x}, \boldsymbol{\xi})-\lambda_{2}\right)} \quad \text { nрu } \alpha \rightarrow \infty .
$$

Следствие 2 выводится из теоремы 1 путем аппроксимации $\chi_{I}$ функциями из класса $C_{0}^{\infty}$. Заметим, что в следствии 2 предполагается, что обе точки $\lambda_{1}, \lambda_{2}$ лежат вне области значений символа $a$. Это приводит к двучленной асимптотике 
функции распределения. Если это ограничение на $\lambda_{1}, \lambda_{2}$ удалить, то теорема 1 обеспечивает только одночленную асимптотику с остатком вида $o\left(\alpha^{d}\right)$.

2. Интерес к псевдодифференциальным операторам с разрывными символами восходит к классической формуле Сегё для определителя тёплицевой матрицы, см. [16] и [7]. Обширная литература посвящена различным содержательным обобщениям формулы Сегё для одномерного случая, см. книги [1], [12] для ознакомления с предметом, обзор [3] результатов, полученных до 2001 г., и недавнюю работу [2], содержащую последние результаты и дополнительные ссылки. Многомерные обобщения континуального варианта формулы Сегё были получены в работах [11], [17], [18]. В статье [18] рассматривается более общая задача: вместо определителя изучаются подходящие аналитические функции оператора, а также допускаются матричные символы. Для $\Omega=\mathbb{R}^{d}$ и $a(\mathbf{x}, \boldsymbol{\xi})=a(\boldsymbol{\xi})$ показано, что

$$
\operatorname{tr} g\left(T_{\alpha}(a)\right)=\alpha^{d} V_{0}+\alpha^{d-1} V_{1}+o\left(\alpha^{d-1}\right) \quad \text { при } \alpha \rightarrow \infty,
$$

где коэффициенты $V_{0}, V_{1}$ вычислены явно, причем в скалярном случае $V_{0}=$ $\mathfrak{W}_{0}(g(a))$. Член порядка $\alpha^{d-2}$ был найден в работе [13] при дополнительных условиях гладкости на границу $\partial \Lambda$.

Когда $\Lambda \neq \mathbb{R}^{d}$ и $\Omega \neq \mathbb{R}^{d}$, т. е. у символа есть скачки по обеим переменным, формула (7) неприменима. Вместо нее Видом в работе [19] предложил в качестве гипотезы формулу (6). Эта гипотеза была доказана в [19] для $d=1$. В многомерном случае асимптотика (6) была обоснована в [20] при условии, что одна из областей - полупространство, а функция $g$ аналитична в круге достаточно большого радиуса. После работы [20] было лишь небольшое число публикаций с частичными результатами. С помощью абстрактного варианта формулы Сегё с оценкой остатка (см. [9], [10]) в работах [4], [5] была установлена точная оценка

$$
\operatorname{tr} g\left(T_{\alpha}(a)\right)-\alpha^{d} \mathfrak{W}_{0}(g(a))=O\left(\alpha^{d-1} \log \alpha\right) .
$$

В статье [6] было замечено, что формула (6) связана с поведением энтропии сцепленности свободных фермионов в основном состоянии. Как объяснено в [6], изучаемая энтропия находится по формуле $\operatorname{tr} h\left(\chi_{\Lambda} P_{\Omega, \alpha} \chi_{\Lambda}\right)$ с некоторыми ограниченными областями $\Lambda, \Omega$ и функцией

$$
h(t)=-t \log t-(1-t) \log (1-t), \quad t \in(0,1) .
$$

Поскольку $h(0)=h(1)=0$, главный член в (6), т. е. $\mathfrak{W}_{0}(h(1))$, обращается в нуль, и формула (6) дает $\alpha^{d-1} \log \alpha$-асимптотику следа, которая совпадает с ожидаемым квазиклассическим ростом энтропии. Однако формула (6) неприменима к негладким функциям и, в частности, к функции (9). С другой стороны, в недавней статье [8] асимптотика (6) была доказана для квадратичной функции $g$. Если $g(t)=t-t^{2}$, то эта формула описывает рост дисперсии числа частиц, что обеспечивает правильную по порядку оценку снизу для энтропии сцепленности.

Ранее в [14] были получены асимптотические формулы для собственных значений и собственных функций оператора $T_{\alpha}(1)$ в случае, когда обе области $\Lambda$ и $\Omega$ - шары в $\mathbb{R}^{d}$. Однако эти результаты не помогают изучать след $\operatorname{tr} g\left(T_{\alpha}(1)\right)$.

3. Полное доказательство теоремы 1 приведено в работе [15]. В настоящей заметке мы даем краткое описание его основных этапов. Доказательство распадается на две неравные части. Решающая, более трудная часть состоит в обосновании асимптотики (6) для полиномиальных функций $g$. 
Теорема 3. Пусть области $\Lambda, \Omega$ и символ а такие же, как в теореме 1. Тогда асимптотика (6) справедлива при $g(t)=t^{p}$, где число $p=1,2, \ldots$ произвольно.

Как только эта теорема доказана, асимптотика замыкается с помощью точной оценки (8), полученной в [4], [5].

Доказательство теоремы 3 включает следующие этапы:

1. Оценки ядерных норм псевдодифференциальных операторов с разрывными символами.

2. Анализ задачи для $d=1$.

3. Геометрическая оценка.

4. Сведе́ние исходной задачи к случаю $d=1$.

Наиболее сложным и важным является шаг 4. Здесь мы делим область $\Lambda$ на граничный слой, который дает вклад в первый и во второй члены асимптотики (6), и внутреннюю часть, которая влияет только на главный член. Затем мы строим две функции (обозначаемые через $q^{\downarrow}=q^{\downarrow}(\mathbf{x})$ и $q^{\uparrow}=q^{\uparrow}(\mathbf{x})$ соответственно), образующие разбиение единицы, подчиненное соответствующему покрытию области. Интересно отметить, что толщина граничного слоя не зависит от $\alpha$. Асимптотика $\operatorname{tr}\left(q^{\uparrow} g\left(T_{\alpha}\right), \alpha \rightarrow \infty\right.$, не «чувствует» границы, и относительно стандартные квазиклассические соображения приводят к формуле

$$
\operatorname{tr}\left(q^{\uparrow} g\left(T_{\alpha}\right)\right)=\alpha^{d} \mathfrak{W}_{0}\left(q^{\uparrow} g(a)\right)+O\left(\alpha^{d-1}\right), \quad \alpha \rightarrow \infty .
$$

Чтобы справиться со следом $\operatorname{tr}\left(q^{\downarrow} g\left(T_{\alpha}\right)\right)$, мы строим покрытие граничного слоя открытыми множествами специальной формы. Для каждого из этих множеств граница $\partial \Lambda$ аппроксимируется касательной гиперплоскостью, что позволяет рассмотреть оператор $T_{\alpha}$ как псевдодифференциальный оператор на граничной гиперплоскости, символ которого является оператором того же типа, но в размерности один. Эта редукция приводит к шагу 2 нашего плана. Одномерная ситуация была изучена в работе [19]. Хотя ее результаты не применимы напрямую, предложенный в ней метод позволяет вывести требуемую асимптотику. Это приводит к формуле

$\operatorname{tr}\left(q^{\downarrow} g\left(T_{\alpha}\right)\right)=\alpha^{d} \mathfrak{W}_{0}\left(q^{\downarrow} g(a)\right)+\alpha^{d-1} \log \alpha \mathfrak{W}_{1}(\mathfrak{A}(g ; a))+o\left(\alpha^{d-1} \log \alpha\right), \quad \alpha \rightarrow \infty$.

Складывая (10) и (11), получаем (6).

Сведение к одномерной задаче опирается на результат геометрического характера, который стоит в приведенном списке под номером 3. Он состоит в следующем. Представляя $\boldsymbol{\xi} \in \mathbb{R}^{d}$ в виде $\boldsymbol{\xi}=(\hat{\boldsymbol{\xi}}, t)$, где $\hat{\boldsymbol{\xi}}=\left(\xi_{1}, \ldots, \xi_{d-1}\right), t=\xi_{d}$, введем для каждого $\hat{\xi} \in \mathbb{R}^{d-1}$ множество

$$
\Omega(\hat{\boldsymbol{\xi}})=\{t:(\hat{\boldsymbol{\xi}}, t) \in \Omega\} \subset \mathbb{R} .
$$

Если оно непусто, это счетное объединение открытых интервалов, длины которых мы обозначаем через $\rho_{j}=\rho_{j}(\hat{\boldsymbol{\xi}}), j=1,2, \ldots$ В случае если $\Omega(\hat{\boldsymbol{\xi}})$ пусто, положим $\rho_{j}(\hat{\boldsymbol{\xi}})=\infty$. Решающее наблюдение состоит в том, что при достаточной гладкости границы $\partial \Omega$ функция

$$
m_{\delta}(\hat{\boldsymbol{\xi}})=\sum_{j} \rho_{j}(\hat{\boldsymbol{\xi}})^{-\delta}
$$

принадлежит $L^{1}\left(\mathbb{R}^{d-1}\right)$ для любого $\delta \in(0,2)$. 
C технической точки зрения шаги 2 и 4 основаны на оценках ядерных норм, полученных на первом шаге. Здесь нам приходится строить квазиклассическое исчисление псевдодифференциальных операторов с разрывными символами с явным контролем их свойств относительно масштабных преобразований.

Уместно сравнить наше доказательство с приведенным в работе Видома [20], в которой асимптотика (6) была обоснована в случае, когда $\Lambda$ (или $\Omega$ ) - полупространство. Наш план совпадает с планом Видома в [20]. С другой стороны, относительный вес четырех составляющих меняется. Так, если $\Lambda$ - полупространство, то сведение к одномерному случаю (т. е. шаг 4) происходит практически мгновенно, тогда как в настоящей работе в случае произвольных $\Lambda$ такое сведение вызывает серьезные затруднения. Далее, в [20] на шаге 3 было достаточно получить геометрическую оценку для $\delta=1$. Нам такая оценка необходима для $\delta>1$. Кроме того, вывод оценок ядерных норм в [20] опирается на предположение, что $\Lambda$ - полупространство, а этого, очевидно, недостаточно для наших рассмотрений. Что касается асимптотики для одномерного случая (т. е. шага 2), то наши оценки, по-видимому, более информативны, так как помимо зависимости от параметра $\alpha$ они позволяют следить за зависимостью от масштабных преобразований.

\section{ЛитеРАТУРА}

[1] A. Böttcher, B. Silbermann, Analysis of Toeplitz operators, Springer Monographs in Math., Springer-Verlag, Berlin, 2006. [2] P. Deift, A. Its, I. Krasovsky, http://arxiv.org/abs/0905.0443v2. [3] T. Ehrhardt, in: Recent Advances in Operator Theory (Groningen, 1998), Oper. Theory Adv. Appl., vol. 124, Birkhäuser, Basel, 2001, 217-241. [4] D. Gioev, Generalizations of Szegö Limit Theorem: Higher Order Terms and Discontinuous Symbols, PhD Thesis, Dept. of Math., Royal Inst. of Technology (KTH), Stockholm, 2001. [5] D. Gioev, Int. Math. Res. Not., 2006, Art. ID 95181. [6] D. Gioev, I. Klich, Phys. Rev. Lett., 96:10 (2006), id. 100503. [7] U. Grenander, G. Szegő, Toeplitz Forms and Their Applications, Univ. of California Press, Berkeley-Los Angeles, 1958. [8] R. C. Helling, H. Leschke, W. L. Spitzer, http://dx.doi.org/10.1093/imrn/rnq085. [9] A. Laptev, Yu. Safarov, in: Contemporary Mathematical Physics, Amer. Math. Soc. Transl. Ser. 2, vol. 175, Amer. Math. Soc., Providence, RI, 1996, 69-79. [10] A. Laptev, Yu. Safarov, J. Funct. Anal., 138:2 (1996), 544-559. [11] И. Ю. Линник, Изв. АН СССР, сер. матем., 39:6 (1975), 1393-1403. [12] N. K. Nikolski, Operators, Functions, and Systems: an Easy Reading. Vol. 1. Hardy, Hankel, and Toeplitz, Mathematical Surveys and Monographs, vol. 92, Amer. Math. Soc., Providence, RI, 2002. [13] R. Roccaforte, Trans. Amer. Math. Soc., 285:2 (1984), 581-602. [14] D. Slepian, Bell System Tech. J., 43 (1964), 3009-3057. [15] A. V. Sobolev, http://arxiv.org/1004.2576v1. [16] G. Szegö, Meddel. Lunds Univ. Mat. Sem., 1952, Suppl. vol. M. Riesz, 228-238. [17] H. Widom, Trans. Amer. Math. Soc., 94:1 (1960), 170-180. [18] H. Widom, J. Funct. Anal., 39 (1980), 182-198. [19] H. Widom, in: Toeplitz Centennial (Tel Aviv, 1981), Oper. Theory Adv. Appl., vol. 4, Birkhäuser, Basel-Boston, Mass., 1982, 477-500. [20] H. Widom, J. Funct. Anal., 88:1 (1990), 166-193. 